\title{
Implementation of smartphone applications supporting end-to-end communication
}

\author{
Katsuhiro Naito \\ Aichi Institute of Technology \\ 1247 Yachigusa, Yakusa, \\ Toyota, Japan \\ naito@pluslab.org
}

\author{
Fumihito Sugihara \\ Mie University \\ 1577 Kurimamachiya, \\ Tsu, Japan \\ fsugihara@com.elec.mie- \\ u.ac.jp
}

\author{
Hiroshi Nodo \\ Meijo University \\ 1-501 Siogamahuchi, \\ Tenpaku,Nagoya, Japan \\ hiroshi.noudou@wata- \\ lab.meijo-u.ac.jp
}

\begin{abstract}
This paper proposes a new application-based end-to-end communication platform for smartphones. We have implemented a "Network Traversal with Mobility" (NTMobile) function, which is an IP mobility protocol developed by the authors, as an application library, and extended it suitable for smartphone devices. To the best of our knowledge, this is globally the first attempt to realize full connectivity and IP mobility in the application layer on smartphone OSs. By using our developed application platform, iOS and Android devices can start communication with each other in any combinations of IPv4 private networks, IPv4 global networks, and IPv6 networks, and they can change their access networks with maintaining the communication.
\end{abstract}

\section{Categories and Subject Descriptors}

C.2.5 [Computer-Communication Networks]: Local and Wide-Area Networks - Internet; C.5.3 [Computer System Implementation]: Microcomputers-Portable devices

\section{General Terms}

Design, Experimentation, Performance, Verification

\section{Keywords}

iOS, Android, IP Mobility platform, IPv4/IPv6, Application layer

\section{INTRODUCTION}

A lot of smartphone applications have been released in recent years. However, servers and network resources are now required to deal with a huge traffic volume in proportion to the spread of applications, as most applications employ the conventional client-server model due to the NAT traversal problem in practical networks. Thus, it is quite useful to find a solution to alleviate the load on servers and use network resources effectively towards the future. Relying upon the IP mobility mechanism is one of the solu-

Permission to make digital or hard copies of all or part of this work for personal or classroom use is granted without fee provided that copies are not made or distributed for profit or commercial advantage and that copies bear this notice and the full citation on the first page. To copy otherwise, to republish, to post on servers or to redistribute to lists, requires prior specific permission and/or a fee. MOBIQUITOUS 2014, December 02-05, London, Great Britain Copyright (c) 2014 ICST 978-1-63190-039-6 DOI 10.4108/icst.mobiquitous.2014.257908

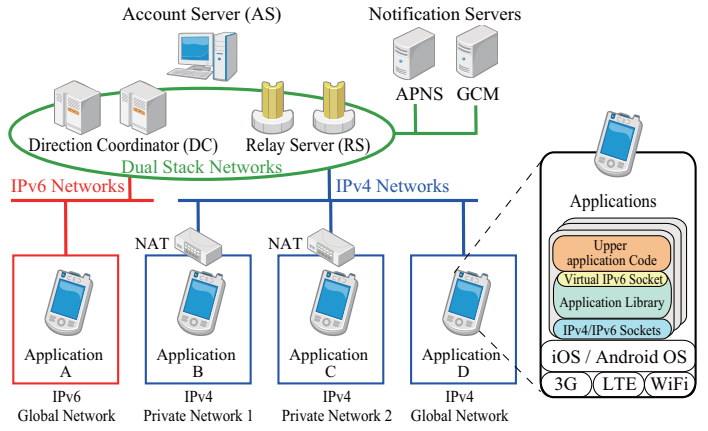

Figure 1: End-to-end communication platform.

tions to the problem. The IP mobility mechanism can provide end-to-end communication services even when IP addresses change due to the clients' relocation or the switching of access networks $[1,2]$. This paper describes a prototype application which supports end-to-end communication for Android and iOS. Our implementation enables end-to-end communication among IPv4 global networks, IPv4 private networks and IPv6 networks, and IP mobility, as well as secure communications with shared keys through the account authentication. Software developers can easily use our proposed application library in their applications without the necessity of obtaining a root authority. The library employs NTMobile $[3,4]$ as the core IP mobility function.

\section{END-TO-END COMMUNICATION PLAT- FORM}

\subsection{System model}

Fig. 1 shows the system model of our proposed end-to-end communication platform for smartphone applications. It enables end-to-end secure communication, accessibility behind NAT routers and inter-connectivity between IPv4 and IPv6 networks. The system consists of an account server (AS), and a number of sets of a direction coordinator (DC) and relay servers (RSs). All DCs are linked to AS, and RSs are linked to their DC. The system can be managed hierarchically and has a high scalability. In addition, DC has notification services such as Apple Push Notification Service (APNS) and Google Cloud Messaging for Android (GCM) to notify the start of communication to the application library. AS manages user information in the platform. DC manages the assignment of virtual IP addresses and directs end nodes to create a tunnel route. RS relays packets when 


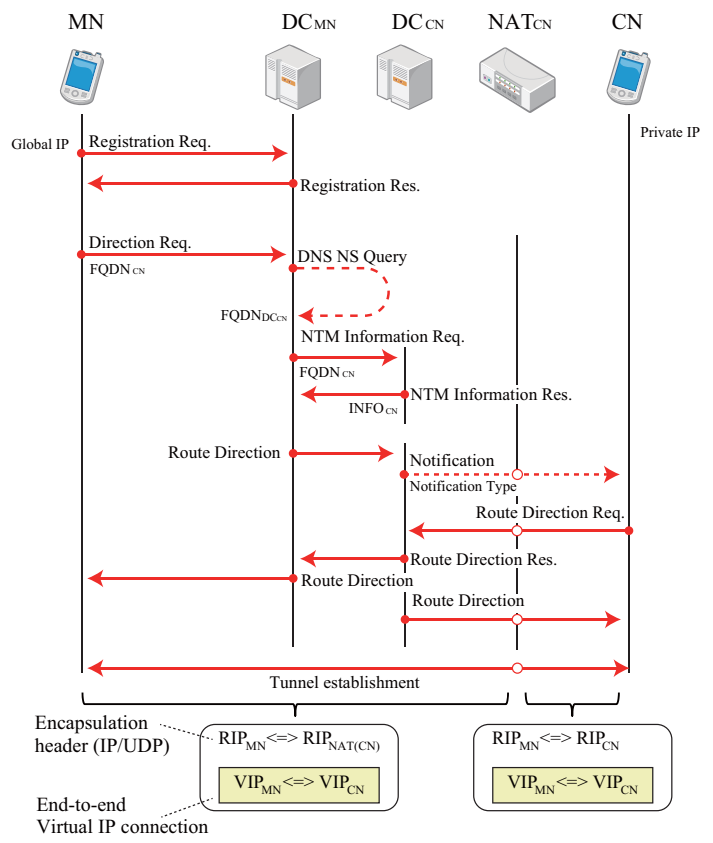

Figure 2: Signaling process.

the route cannot be created directly; e.g., when both devices are connected in private networks behind NAT routers, or each device is connected to a different address family, that is IPv4 and IPv6. The application library provides NTMobile functions to its upper applications. It employs real IPv4 and real IPv6 sockets for the sake of exchanging UDP tunneling messages in NTMobile, and provides a virtual IPv6 socket for upper applications. Therefore, software developers can implement their applications by using general BSD sockets. Additionally, it can be installed with the user authority for smartphone applications.

\subsection{Signaling process}

Fig. 2 shows the signaling process to create a tunnel between application libraries. The application library in MN registers its real IP address to its $\mathrm{DC}_{\mathrm{MN}}$ when an application is launched and obtains a unique virtual IPv6 address from $\mathrm{DC}_{\mathrm{MN}}$. The virtual IPv6 address is used to establish a connection between upper applications. The application library in MN initiates the following NTMobile signaling when a new socket is opened by the application. The first process is to send a Direction Request message to $\mathrm{DC}_{\mathrm{MN}}$, requesting for a tunnel creation between $\mathrm{MN}$ and $\mathrm{CN}$. $\mathrm{DC}_{\mathrm{MN}}$ finds the IP address of $\mathrm{DC}_{\mathrm{CN}}$ with the DNS name resolution mechanism. Then, $\mathrm{DC}_{\mathrm{MN}}$ obtains the address information of $\mathrm{CN}$ from $\mathrm{DC}_{\mathrm{CN}}$ by exchanging NTM Information Request and Response messages. Then, $\mathrm{DC}_{\mathrm{MN}}$ determines how to create the optimal tunnel based on the IP address information of $\mathrm{MN}$ and $\mathrm{CN}$, and directs $\mathrm{MN}$ and $\mathrm{CN}$ by Route Direction messages to create the tunnel. In the case of Fig. 2, DC $\mathrm{MN}_{\mathrm{N}}$ decides to create a direct tunnel because MN has a global IP address. Then, $\mathrm{DC}_{\mathrm{MN}}$ requests $\mathrm{DC}_{\mathrm{CN}}$ to create a tunnel between MN and CN by sending the Route Direction message. $\mathrm{DC}_{\mathrm{CN}}$ uses APNS or GCM to notify the application library in CN. The application library in CN replies the Route Direction Request message so that it obtains the Route Direction message. After receiving the Route Direction Response message, $\mathrm{DC}_{\mathrm{MN}}$ sends the Route Direction message to MN.

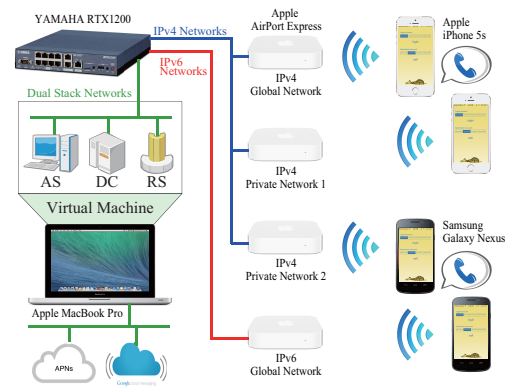

Figure 3: Prototype implementation.

Then, MN and CN exchange Tunnel Request and Response messages directly with each other to create the direct tunnel between them.

\section{IMPLEMENTATION}

We have developed a prototype application for free voice communications, by applying our proposed platform in iOS and Android OS. Fig. 3 shows the configuration of our implementation. Our developed applications are installed in these devices with the user authority just like usual smartphone applications. The application can start free voice communication with each other in any network combinations, and it can change its access networks with maintaining the communication.

\section{CONCLUSIONS}

This paper has proposed a prototype application which supports end-to-end communication. The application is the first fundamental prototype employing IP mobility in an application layer. The advantages of the proposal are that software developers can implement their applications by using general BSD sockets and our IP mobility function can be installed with the user authority for smartphone applications.

\section{Acknowledgment}

This work is supported in part by the Grant-in-Aid for Scientific Research (C)(26330103), Japan Society for the Promotion of Science (JSPS) and the Integration research for agriculture and interdisciplinary fields, Ministry of Agriculture, Forestry and Fisheries, Japan.

\section{ADDITIONAL AUTHORS}

Additional authors: Masanori Kako (Meijo University), Tatsuya Hirose (Meijo University), Hidekazu Suzuki (Meijo University), Akira Watanabe (Meijo University), Kazuo Mori (Mie University and Hideo Kobayashi (Mie University).

\section{REFERENCES}

[1] D. Le, X. Fu and D. Hogrere, "A Review of Mobility Support Paradigms for the Internet," IEEE Communications surveys, 1st quarter 2006, Volume 8, No. 1, 2006.

[2] C. Perkins, "IP Mobility Support for IPv4, Revised," RFC 5944, IETF (2010).

[3] K. Naito et al. Proposal of Seamless IP Mobility Schemes: Network Traversal with Mobility (NTMobile). In Proc. IEEE GLOBECOM 2012, Dec. 2012.

[4] project NTMobile. http://www.ntmobile.net 